\title{
Modification of AQI as an Effective Representation of Air Quality by Considering Weighting Factors
}

\author{
Li Wei Lai ${ }^{1, ~ *, ~ W a n ~ L i ~ C h e n g ~}{ }^{2}$ \\ ${ }^{1}$ Centre for General Education, National Taipei University of Business, Taipei, Taiwan, Republic of China \\ ${ }^{2}$ Green Science Technology Co. Ltd., Taichung, Taiwan, Republic of China
}

\section{Email address:}

liweilai@ntub.edu.tw(Li Wei Lai),wlcheng0606@gmail.com (Wan Li Cheng)

${ }^{*}$ Corresponding author

\section{To cite this article:}

Li Wei Lai, Wan Li Cheng. Modification of AQI as an Effective Representation of Air Quality by Considering Weighting Factors. American Journal of Environmental Science and Engineering. Vol. 4, No. 1, 2020, pp. 7-12. doi: 10.11648/j.ajese.20200401.12

Received: April 18, 2020; Accepted: May 9, 2020; Published: May 28, 2020

\begin{abstract}
Controversy persists because residents in regions where air quality is poor are always dissatisfied with the presentation of the air quality index (AQI). To make management policies acceptable, it needs to be determined whether adding the various weighting factors can make the AQI more reasonable and practical. The authors selected three indices i.e. AQI, revised air quality index (RAQI), and the air-dispersion AQI (ADRAQI) to compare their results in different atmospheric situations and to determine whether the AQI was made more reliable by adding various weighting factors such as multi-air pollutants and air dispersion derived from the mean function and an entropy function. Results show in comparison to AQI, the RAQI and ADRAQI have greater values in the multi-air pollutant and poor dispersion events, leading to a great difference between single air pollutant and multi-air pollutant events. The eclipsed phenomena in the AQI for the means of diseases related to air pollution such as acute atopic conjunctivitis, other chronic allergic conjunctivitis, other atopic dermatitis and related conditions, contact dermatitis and other eczema, and unspecified causes clearly appear. The findings suggest that the representation of AQI can be modified by considering the weighting factors of multiple air pollutants along with air dispersion; these can easily be applied to similar regions elsewhere.
\end{abstract}

Keywords: Air Quality Index, Air-dispersion, Multi-air Pollutant, Weighting Actors, Diseases Related to Air Pollution

\section{Introduction}

Prior to using the AQI [1], since November 1994, the Taiwan Environmental Protection Administration (Taiwan EPA) used the pollution standard index (PSI) from the U.S. EPA when presenting air quality to the public on its website [2]. In December 2016, the Taiwan EPA started using the AQI which replaced the PSI and $\mathrm{PM}_{2.5}$ indices [3]. However, there has so far been some dissatisfaction among the Taiwanese people with regard to the reliability of the presentation of the AQI, even though the Taiwan EPA has attempted to offer better air quality information to residents. First, the use of an entirely foreign index without considering geographical conditions in the estimation of the index for the local area was considered as unsuitable. Second, the AQI selects the maximum sub-index from the concentrations of air pollutants. Therefore, it fails to show the influence of air quality on public health when there is pollution from multiple air pollutants [4]. Third, the breakpoints of the AQI are mainly determined by the symptoms or diseases associated with respiratory and cardiovascular systems [5] and may underestimate the real health effects of the air quality, especially in areas where this is poor. For example, people may feel uncomfortable because of the effect of air pollution on their skin [6, 7] or in their eyes [8-10].

By considering different weighting factors, such as multi-pollutant using the background arithmetic mean and Shannon's entropy, Cheng et al. [11] modified the daily AQI, naming it the revised air quality index (RAQI) based on the AQI. Compared with AQI, RAQI considers the influence of multi-air pollutants, responding to a greater range of the index scale, while offering greater accuracy with respect to the unhealthy daily rate [12]. To clarify the issues raised by the public, we selected three indices that are based on the maximum sub-index, i.e. AQI, revised air quality index 
(RAQI), and the air-dispersion AQI (ADRAQI) to compare their results in different atmospheric situations.

This study aims to determine whether adding various weighting factors such as multiple air pollutants and air dispersion derived from the mean function and an entropy function made the AQI more reasonable and practical.

\section{Materials and Methods}

\subsection{Study Area}

The weather in Taiwan is dominated by north-easterly and south-westerly monsoons in winter and summer, respectively, each lasting half a year [13]. The Kaoping region of the study area, including Kaohsiung City and Northern Pingtung County, is in southwestern Taiwan (Figure 1). In the winter, i.e. from October to April, this area is on the leeward side of the Central Mountain Range for the north-easterly monsoon; therefore, air dispersion is very poor during this period. In the region, when $\mathrm{AQI} \geq 151$, the major air pollution triggers are $\mathrm{PM}_{2.5}$ and $\mathrm{PM}_{2.5} \& \mathrm{O}_{3}$ events.

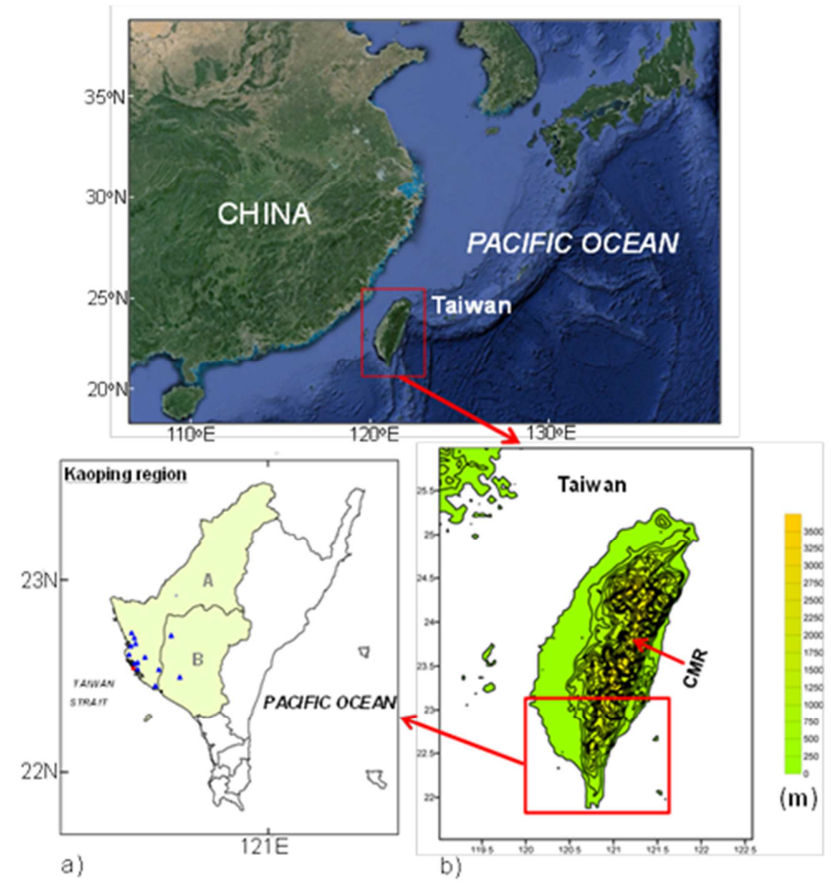

Figure 1. Study area: a) location of Taiwan Central Weather Bureau (CWB) station and Environmental Protection Agency (EPA) in the Kaoping region; b) terrain of Taiwan. A: Kaohsiung City. B: Northern Pingtung County. Blue triangles: 14 Taiwan EPA air-quality monitoring sites. Red square: the Kaohsiung meteorological station. CMR: Central Mountain Range. The satellite figure was from https://earth.google.com/web/@30.95252676,123.29904618,-3869.8010261 8a,4772806.30663455d,35y,-0h,0t,0r (Google Earth).

\subsection{Data}

The study period ranged from 1 January 2006 to 31 December 2016. The hourly concentrations of $\mathrm{PM}_{10}, \mathrm{PM}_{2.5}, \mathrm{O}_{3}$, $\mathrm{CO}, \mathrm{NO}_{2}$ and $\mathrm{SO}_{2}$ in the 14 air quality monitoring stations were obtained from the Taiwan EPA. The hourly surface air temperature, dew temperature, and wind speed at the Kaohsiung weather station were obtained from the Taiwan Central Weather Bureau (CWB). In this study, the number of admissions during the period ranging from 2006-2015 was classified according to the International Classification of Disease, 9th edition (ICD-9-CM), and the data from 2016 were based on the International Classification of Disease, 10th edition (ICD-10-CM). The daily number of admissions of acute atopic conjunctivitis (ICD-9-CM 372.05; ICD-10-CM H10.10 H10.11 H10.12 H10.13), other chronic allergic conjunctivitis (ICD-9-CM 372.14; ICD-10-CM H10.411 H10.412 H10.413 H10.419 H10.45), circulatory system diseases (ICD-9-CM 390-459; ICD-10-CM I00-I99.9), respiratory system diseases (ICD-9-CM 460-519; ICD-10-CM J00-J98.9), influenza with pneumonia (ICD-9-CM 487; ICD-10-CM J09. X1-J11.89); asthma (ICD-9-CM 493; ICD-10-CM J45.20-J45.901), other atopic dermatitis and related conditions (ICD-9-CM 691.8; ICD-10-CM L20.0-L20.9), contact dermatitis and other eczema, and unspecified cause (ICD-9-CM 692.9; ICD-10-CM L23.9 L24.9 L25.9 L30.0 L30.2 L30.8 L30.9) was obtained from the Applied Health Research Data Integration Service from the National Health Insurance Administration (NHIA), Ministry of Health and Welfare. To manage the influenza effect, the admissions of influenza with pneumonia (ICD-9-CM 487; ICD-10-CM J09. X1-J11.89) were excluded in the admissions of respiratory system diseases (ICD-9-CM 460-519; ICD-10-CM J00-J98.9). Private data such as admitted patient's identity numbers and addresses are unavailable; therefore, it was not possible to prevent the occurrence of bias, such as whether the admission was a Kaoping resident or whether the patient was a readmission which was unavoidable.

\subsection{Air Quality Indices}

The daily AQI was derived using the maximum sub-index, which was obtained from the measurement of the main air pollutant concentrations (Table 1), such as $\mathrm{PM}_{10}$ (an aerodynamic diameter of $\leq 10 \mu \mathrm{m}$ ), $\mathrm{PM}_{2.5}$ (an aerodynamic diameter of $<2.5 \mu \mathrm{m})$, ozone $\left(\mathrm{O}_{3}\right)$, carbon monoxide $(\mathrm{CO})$, sulphur dioxide $\left(\mathrm{SO}_{2}\right)$, and nitrogen dioxide $\left(\mathrm{NO}_{2}\right)$ [3]. The scale of the AQI system uses 50, 100, 150, 200, 300, and 500, according to the National Ambient Air Quality Standards (NAAQS) [5]. The Taiwan EPA defined the breakpoint concentrations based on NAAQS and other epidemiological studies.

The term on the right side in Eq. (1), $\operatorname{Max}_{\text {daily }}\left[\mathrm{S}_{1}, \mathrm{~S}_{2}, \ldots\right.$, $\left.\mathrm{S}_{n}\right]$, is the maximum sub-index $\left(\mathrm{S}_{1}, \mathrm{~S}_{2}, \ldots, \mathrm{S}_{n}\right)$ of the measurement from the daily air pollutant concentration, while $n=6$ for AQI.

$$
\mathrm{AQI}=\operatorname{Max}_{\text {daily }}\left[\mathrm{S}_{1}, \mathrm{~S}_{2}, \ldots, \mathrm{S}_{n}\right]
$$

The ADRAQI derived from the two weighting factors $\left(\mathrm{WF}_{1}\right.$ and $\left.\mathrm{WF}_{2}\right)$, with a mean function and an entropy function, is as shown in Eq. (2). 
Table 1. Breakpoints for AQI (Taiwan EPA, 2016) in this study.

\begin{tabular}{lllllllll}
\hline AQI & $\mathbf{O}_{3,8-h r}(\mathbf{p p m})$ & $\mathbf{O}_{3,1-h r}(\mathbf{p p m})$ & $\mathbf{P M}_{\mathbf{1 0}}\left(\boldsymbol{\mu g} / \mathbf{m}^{3}\right)$ & $\mathbf{P M}_{2.5}\left(\boldsymbol{\mu g} / \mathbf{m}^{3}\right)$ & $\mathbf{C O}(\mathbf{p p m})$ & $\mathbf{S O}_{2}(\mathbf{p p m})$ & $\mathbf{N O}_{2}(\mathbf{p p m})$ & $\mathbf{E f f e c t ~ o n ~ h e a l t h ~}$ \\
\hline $0-50$ & 0.054 & - & 54 & 15.4 & 4.4 & 0.035 & 0.053 & Good \\
$51-100$ & 0.070 & - & 125 & 35.4 & 9.4 & 0.075 & 0.1 & Moderate \\
$101-150$ & 0.085 & 0.164 & 254 & 54.4 & 12.4 & 0.185 & 0.36 & Unhealthy for \\
$151-200$ & 0.105 & 0.204 & 354 & 150.4 & 15.4 & 0.304 & 0.649 & sensitive groups \\
$201-300$ & 0.200 & 0.404 & 424 & 250.4 & 30.4 & 0.604 & 1.249 & Vnhealthy \\
$301-500$ & & 0.604 & 604 & 500.4 & 50.4 & 1.004 & 2.049 & Hazardous \\
\hline
\end{tabular}

$$
\begin{gathered}
\text { ADRAQI }=\mathrm{AQI} \times \mathrm{WF}_{1} \times \mathrm{WF}_{2} \\
\mathrm{WF}_{1}=\frac{\frac{1}{n} \sum_{i=1}^{n}\left[S_{i}\right]}{\frac{1}{m} \sum_{j=1}^{m}\left[\frac{1}{n} \sum_{i=1}^{n} S_{i, j}\right]} \times \frac{\frac{1}{m} \sum_{j=1}^{m}\left\{\text { Entropy }_{j}\left[\text { Max }_{\text {hourly }}\left[S_{1}, S_{2}, \ldots, S_{n}\right]\right]\right\}}{\text { Entropy }\left[\operatorname{Max}_{\text {hourly }}\left[S_{1}, S_{2}, \ldots, S_{n}\right]\right]} \\
\mathrm{WF}_{2}=\frac{\frac{1}{m} \sum_{j=1}^{m} \text { PBLH }_{j}}{\text { Mean }\left[\text { PBLH } \text { hourly }_{\text {f }}\right]} \times \frac{\text { Entropy }[\text { PBLH }]}{\frac{1}{m} \sum_{j=1}^{m}\left\{\text { Entropy }_{j}[\text { PBLH }]\right\}}
\end{gathered}
$$

In this study, the Nozaki planetary boundary layer height $(P B L H)$ was calculated as shown in Eq. (5) and Eq. (6) [14], subsequently being referred to as a weighting factor of air dispersion ( $\mathrm{WF}_{2}$; Eq. (4)).

$$
P B L H=\frac{121}{6}(6-S)\left(T-T_{d}\right)+\frac{0.169 S\left(W S_{h}+0.257\right)}{12 f \ln (h / L)}
$$

where $h$ is the altitude of the monitoring site, $L$ is the roughness of surface, $S$ is the stable parameter, $T-T_{d}$ is the difference between the surface air temperature and the dew-point temperature, $W S_{h}$ is the mean wind speed at an altitude of $h$.

$$
f=2 \Omega \sin \theta
$$

where $f$ is the Earth's rotational parameter, $\Omega$ is the Earth's rotational speed, and is the latitude of the monitoring site.

The second term in Eq. (2) is the first weighting factor $\left(\mathrm{WF}_{1}\right.$, (Eq. (3)), where the first operator is the background arithmetic mean, and the second operator is the background arithmetic mean entropy. This term considers the presence of multi-air pollutants, and can reform the deviation and divergence in the calculation of AQI, thus creating the RAQI $\left(\mathrm{RAQI}=\mathrm{AQI} \times \mathrm{WF}_{1}\right.$; $[11,12])$, while improving the ambiguous and eclipsed problems of the air-pollution index [15].

Wind speed and air stability influence the dispersion of air pollutants. The mixing height, which shows the limited height of vertical air movement, is related to air quality $[16,17]$. In this study, to consider local geographical features while determining the air quality accurately, it is important to consider the addition of another weighting factor to the AQI. The third term in Eq. (2) is the second weighting factor $\left(\mathrm{WF}_{2}\right.$, Eq. (4)), with the first operator, which indicates the background arithmetic mean, being composed of the average of the sum of daily mixing height as the numerator (where $\mathrm{m}=$ the number of days from 2006 to 2016) and the daily average of the mixing height as the denominator. The second operator indicates the background arithmetic mean entropy, where the numerator is the entropy function of the daily average of the mixing height, and the denominator is the average of the sum of the entropy function of the daily average of the mixing height. For $\mathrm{WF}_{2}$, when the first operator is greater, the seconder operator is smaller, and vice versa. Therefore, the greater value of $\mathrm{WF}_{2}$ represents the influence of poor air dispersion, and vice versa. For example, by considering $\mathrm{WF}_{1}$ and $\mathrm{WF}_{2}, 83$ daily ADRAQI values were greater than the daily RAQI, and 52 daily ADRAQI values were smaller than the daily RAQI from 2006 to 2016 (Figure 2 (a) and (b)). This term considers local geographical features, such as the altitude of the monitoring site and the roughness of the surface. It also includes thermal and dynamic features such as the temperature and wind speed, respectively, which are important factors that may influence the air dispersion.

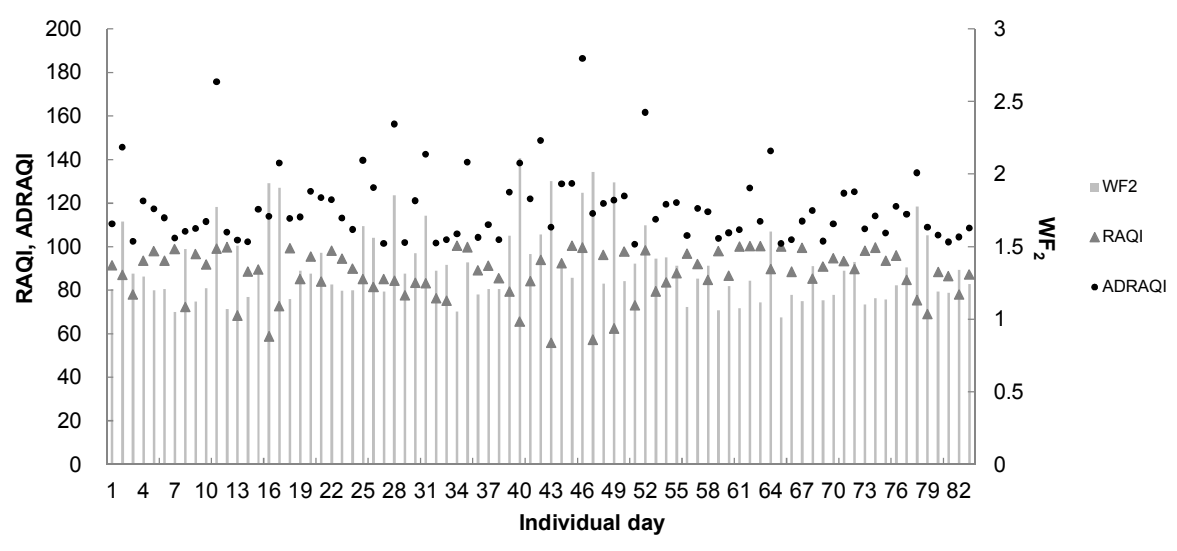




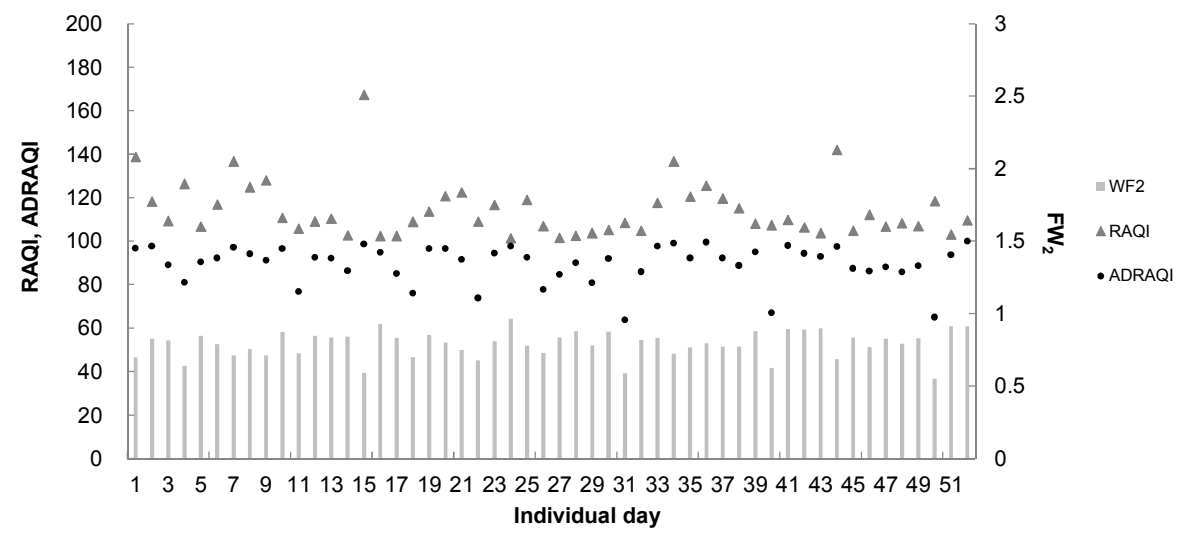

b)

Figure 2. Relationship between RAQI, ADRAQI and the weighting factor of air dispersion (WF 2 from 2006 to 2016: a) ADRAQI is greater than RAQI; b) $A D R A Q I$ is lower than RAQI. RAQI: $A Q I \times W F_{1}$. ADRAQI: RAQI $\times W F_{2}$.

\section{Results}

\subsection{Presentation of the Reality of Air Quality Indices}

The distribution of the frequency of daily AQI, RAQI, and ADRAQI shows that $34.2 \%$ of the RAQI and $34.8 \%$ of the ADRAQI were $\geq 151$. However, less than $25 \%$ of AQI was $\geq$ 151. This suggests that the AQI is more relaxed than RAQI and ADRAQI.

The mean values of AQI, RAQI, and ADRAQI in the $\mathrm{PM}_{2.5}$, $\mathrm{O}_{3}$, and $\mathrm{PM}_{2.5} \& \mathrm{O}_{3}$ events were significantly greater than those in normal conditions $(P<0.05)$ (Table 2). The RAQI and ADRAQI have greater values in the multi-air pollutant and poor dispersion events, leading to a great difference between single air pollutant and multi-air pollutant events. For example, the mean AQI was 163.7 (with a standard deviation of 11.6) for the $\mathrm{O}_{3}$ events and 169.3 (12.9) for the $\mathrm{PM}_{2.5} \& \mathrm{O}_{3}$ events. The mean RAQI was 230.6 (17.2) for the $\mathrm{O}_{3}$ events and 267.4 (31.1) for the $\mathrm{PM}_{2.5} \& \mathrm{O}_{3}$ events, while ADRAQI was 261.1 (31.2) during the $\mathrm{O}_{3}$ events and 295.9 (78.2) during the $\mathrm{PM}_{2.5} \& \mathrm{O}_{3}$ events. It appears that the incorporation of $\mathrm{WF}_{1}$ and $\mathrm{WF}_{2}$ can contribute to improvements in the presentation of the reality of the AQIs.

Table 2. Comparison of mean daily indices and weather parameters for the air-quality conditions in the Kaoping region between October and April from 2006 to 2016.

\begin{tabular}{|c|c|c|c|c|}
\hline Daily indices and weather parameters & Normal $(n=241)$ & $\mathrm{PM}_{2.5}(n=452)$ & $O_{3}(n=22)$ & $\mathrm{O}_{3} \& \mathrm{PM}_{2.5}(n=45)$ \\
\hline AQI & $78.6(16.4)^{\mathrm{a}}$ & $158.5(6.6)^{\mathrm{b}}$ & $163.7(11.6)^{\mathrm{c}, \mathrm{d}}$ & $169.3(12.9)^{\mathrm{d}}$ \\
\hline RAQI & $66.9(22.0)^{\mathrm{a}}$ & $208.7(28.1)^{b}$ & $230.6(17.2)^{\mathrm{c}}$ & $267.4(31.1)^{\mathrm{d}}$ \\
\hline ADRAQI & $69.9(28.8)^{\mathrm{a}}$ & $225.9(70.5)^{b}$ & $261.1(31.2)^{\mathrm{c}}$ & $295.9(78.2)^{\mathrm{d}}$ \\
\hline Mixing height (m) & $4968.5(1395.4)^{\mathrm{a}}$ & $4760.3(1240.6)^{b}$ & $4300.3(908.3)^{\mathrm{b}}$ & $4668.2(1484.7)^{\mathrm{a}, \mathrm{b}}$ \\
\hline Wind speed $(\mathrm{m} / \mathrm{s})$ & $2.1(0.6)^{\mathrm{a}}$ & $1.9(0.5)^{\mathrm{b}}$ & $1.7(0.2)^{\mathrm{b}}$ & $1.7(0.3)^{\mathrm{b}}$ \\
\hline
\end{tabular}

Mean with the different letter are significantly different at the 0.05 level. ( ) indicates the standard deviation.

\subsection{Eclipsed and Ambiguous Phenomena in AQI}

Table 3 presents the comparison of the number of admissions for RAQI between categories $\mathrm{A}$ and $\mathrm{B}$, and for ADRAQI between categories $\mathrm{C}$ and $\mathrm{D}$. The null hypothesis $\left(H_{o}\right)$ was that category B (category D) was significantly greater than or equal to category A (category $\mathrm{C}$ ), and the alternative hypothesis $\left(H_{l}\right)$ was that category A (category $\mathrm{C}$ ) was significantly greater than category B (category D).

Table 1 shows the six break points of the AQI categorizing the effects on health. 12 of the daily RAQI data points were characterized as 'unhealthy for sensitive groups' by considering multi-air pollutants from the 'moderate' group, which is called category A, while 71 daily RAQI data points were in the 'moderate' category by considering multi-air pollutants from 'unhealthy for sensitive groups', which is called category B. The mean number of cases of chronic allergic conjunctivitis, atopic dermatitis, and other related conditions in category A are significantly greater than those in category $\mathrm{B}(P<0.05)$. For example, the mean number of admissions due to other chronic allergic conjunctivitis cases was 857.1 day (with a standard deviation of $112.3 /$ day) in category A, and 787.8/day (171.5/day) in category B.

Of the daily ADRAQI readings, 83 were characterized as "unhealthy for sensitive groups" considering poor air dispersion from the "moderate" group, denoted category $\mathrm{C}$, while 52 daily ADRAQI readings were in the "moderate" category considering good air dispersion as "unhealthy for sensitive groups," or category D (Table 3). This means hospital admissions due to acute atopic conjunctivitis, other chronic allergic conjunctivitis, other atopic dermatitis and related conditions, contact dermatitis and other eczema, and unspecified causes in category $\mathrm{C}$ were significantly greater than those in category D $(P<0.05)$. For example, the mean number of acute atopic conjunctivitis cases was 46.2/day (12.9/day) in category $\mathrm{C}$ and $38.4 /$ day (13.7/day) in category $\mathrm{D}$. 
Table 3. Comparison of the number of admissions for RAQI between categories A and B, and for ADRAQI between categories C and D from 2006 to 2016.

\begin{tabular}{lllll}
\hline Diseases & $\mathbf{A}(\boldsymbol{n}=\mathbf{1 2})$ & $\mathbf{B}(\boldsymbol{n}=\mathbf{7 1})$ & $\mathbf{C}(\boldsymbol{n}=\mathbf{8 3})$ & $\mathbf{D}(\boldsymbol{n}=\mathbf{5 2})$ \\
\hline Acute atopic conjunctivitis & $46.5(13.6)$ & $40.4(14.0)$ & $46.2(12.9)^{*}$ & $38.4(13.7)$ \\
Other chronic allergic conjunctivitis & $857.1(112.3)^{*}$ & $787.8(171.5)$ & $870.1(127.9)^{*}$ & $769.8(178.2)$ \\
Circulatory system diseases & $14558.8(2584.5)$ & $14491.2(2121.2)$ & $14625.0(1700.9)$ & $14643.5(1643.8)$ \\
Respiratory system diseases & $37925.2(5282.0)$ & $40172.3(8064.4)$ & $35706.0(5198.0)$ & $40664.7(6354.6)$ \\
Asthma & $832.4(179.7)$ & $859.5(253.8)$ & $791.4(224.6)$ & $801.4(112.0)$ \\
Other atopic dermatitis and related conditions & $349.3(43.9)^{*}$ & $313.6(49.9)$ & $348.0(45.6)^{*}$ & $310.6(47.0)$ \\
Contact dermatitis and other eczema, unspecified cause & $2165.8(196.7)$ & $2063.6(300.7)$ & $2262.8(243.3)^{*}$ & $2023.9(287.1)$ \\
\hline
\end{tabular}

( ) indicates the standard deviation. '“' indicates that the number is clearly larger than the one in categories A and B, and categories $\mathrm{C}$ and $\mathrm{D}$, respectively $(P<$ 0.05 , one-tailed test).

\section{Discussion}

In common real air conditions, more than one air pollutant can contribute to the air-pollution event. Considering multi-air pollutants can lead to a value of the index that is close to the actual air quality because $\mathrm{WF}_{1}$ determines the value of the RAQI under the influence of multi-air pollutants. The breakpoint of 101 is between the 'moderate' category and 'unhealthy for sensitive groups' category (Table 1). When the value of AQI is less than and close to 101, RAQI may fall in the 'unhealthy for sensitive groups' category, based on the greater effect of $\mathrm{WF}_{1}$. Therefore, the eclipsed phenomenon implies that multi-air pollutants exist, suggesting that the actual air quality is worse than the public's expectation of it. On the contrary, when the value of AQI falls in the 'unhealthy for sensitive groups' category, RAQI may fall in the 'moderate' category by considering the reduced effect of $\mathrm{WF}_{1}$. This ambiguous phenomenon implies that there is a small effect of multi-air pollutants, suggesting that the air quality is better than the public's expectation of it.

Air dispersion is related to the concentration of air pollutants in a city $[18,19]$. Air pollutants may accumulate near emission sources under poor air dispersion conditions. Considering that the air dispersion can also shift the index closer to the actual air quality, $\mathrm{WF}_{2}$ determines the ADRAQI under the influence of air dispersion. Therefore, the eclipsed phenomenon implies that the air dispersion is poor, suggesting that the air quality is worse than the public expects. On the contrary, the ambiguous phenomenon implies that the air dispersion is good, suggesting that the air quality is better than the public expects.

\section{Conclusion}

There is still room for the improvement of the AQI. First, the addition of weighting factors of multi-air pollutants can improve the presentation of the reality of the AQIs in areas with pollution due to multiple air pollutants. Second, the eclipsed phenomena of the AQI to the means of acute atopic conjunctivitis, other chronic allergic conjunctivitis, other atopic dermatitis and related conditions, contact dermatitis and other eczema, and unspecified causes can be mitigated by considering multi-air pollutants and air dispersion.

This study suggests that to make efficient management policies, authorities should modify the AQI used for local circumstances. The determination of the breakpoints of a category in an index should not only consider cardiovascular and respiratory system diseases, but also other diseases associated with air pollution.

\section{Acknowledgements}

The authors are grateful to the Taiwan Central Weather Bureau and Taiwan Environmental Protection Administration for providing meteorological and air pollutant concentration data. This study utilized data from the Applied Health Research Data Integration Service from the National Health Insurance Administration, Ministry of Health and Welfare (Registration number B201710130001). The interpretation and conclusion herein do not represent the opinions or influence of the National Health Insurance Administration, Ministry of Health and Welfare. This study has been supported by funding from the Ministry of Science and Technology, Reference No. MOST 106-2111-M-141-001.

\section{References}

[1] United States Environmental Protection Agency (US EPA). Air quality reporting. Federal Register, 1999, 64 (149): 4253042549.

[2] Taiwan Environmental Protection Administration (Taiwan EPA). Air Quality Annual Report of R. O. C. (Taiwan). Taipei, R. O. C.: Taiwan EPA, 2001.

[3] Taiwan Environmental Protection Administration (Taiwan EPA). Air Quality Annual Report of R. O. C. (Taiwan). Taipei, R. O. C.: Taiwan EPA, 2016.

[4] Wark RM, Wong KFV. Methodologies for incorporation of synergistic effects in the calculation of the Pollutant Standards Index. International Journal of Environment and Pollution, 1999, $12 \quad$ (1): 81-90. https://doi.org/10.1504/IJEP.1999.002285.

[5] United States Environmental Protection Agency (US EPA). Technical assistance document for the reporting of daily air quality-the air quality index (AQI). EPA454/B-13-001, 2013. 
[6] Kim HH, Lee CS, Jeon JM, et al. Analysis of the association between air pollution and allergic diseases exposure from nearby sources of ambient air pollution within elementary school zones in four Korean cities. Environmental Science and Pollution Research, 2013, 20 (7): 4831-4846. doi: $10.1007 / \mathrm{s} 11356-012-1358-2$.

[7] Lee C, Kim H, Lim Y, et al. Evaluation of the relationship between allergic diseases in school children at Seoul's roadside elementary schools and air pollution. Atmospheric Pollution Research, 2015, 6 (6): 1004-1012. https://doi.org/10.1016/j.apr.2015.05.006.

[8] Hong J, Zhong T, Li H, et al. Ambient air pollution, weather changes, and outpatient visits for allergic conjunctivitis: A retrospective registry study. Scientific Reports, 2016, 6: 23858. doi: $10.1038 /$ srep23858.

[9] Mimura T, Ichinose T, Yamagami S, et al. Airborne particulate matter $\left(\mathrm{PM}_{2.5}\right)$ and the prevalence of allergic conjunctivitis in Japan. Science of the Total Environment, 2014, 487: 493-499. doi: 10.1016/j.scitotenv.2014.04.057.

[10] Szyszkowicz M, Kousha T, Castner J. Air pollution and emergency department visits for conjunctivitis: A case-crossover study. International Journal of Occupational Medicine and Environmental Health, 2016, 29 (3): 381-393. doi: 10.13075/ijomeh.1896.00442.

[11] Cheng WL, Kuo YC, Lin PL, et al. Revised air quality index derived from an entropy function. Atmospheric Environment, 2004, 38 (3): 383-391. https://doi.org/10.1016/j.atmosenv.2003.10.006.

[12] Cheng WL, Chen YS, Zhang J, et al. Comparison of the revised air quality index with the PSI and AQI indices. Science of the Total Environment, 2007, 382 (2-3): 191-8. doi: 10.1016/j.scitotenv.2007.04.036.
[13] Taiwan Central Weather Bureau (Taiwan CWB). The introduction of characteristics of climate in Taiwan; 2015. Available from: http://www.cwb.gov.tw/V7/climate/climate_info/statistics/stati stics_1_1.html.

[14] Du C, Liu S, Yu X, et al. Urban boundary layer height characteristics and relationship with particulate matter mass concentrations in Xi'an, Central China Aerosol and Air Quality Research, 2013, 13: 1598-1607. doi: 10.4209/aaqr.2012.10.0274.

[15] Swamee PK, Tyagi A. Formation of an air pollution index. Journal of the Air \& Waste Management Association, 1999, 49: 88-91. doi: 10.1080/10473289.1999.10463776.

[16] Pandolfi M, Tobias A, Alastuey A, et al. Effect of atmospheric mixing layer depth variations on urban air quality and daily mortality during Saharan dust outbreaks. Science of the Total Environment, 2014, 494: 283-289. doi: 10.1016/j.scitotenv.2014.07.004.

[17] Prakash D, Payra S, Verma S, et al. Aerosol particle behavior during dust storm and Diwali over an urban location in north western India. Natural Hazards, 2013, 69 (3): 1767-1779. https://doi.org/10.1007/s11069-013-0780-1.

[18] Holzworth GC. Mixing depth, wind speeds and air pollution potential for selected locations in the United States. Journal of Applied Meteorology and Climatology, 1967, 6: 1039-1044. https://doi.org/10.1175/1520-0450(1967)006<1039:MDWSA $\mathrm{A}>2$.0.CO;2.

[19] Mayer H. Air pollution in cities. Atmospheric Environment, 1999, 33: 4029-4037. http://dx.doi.org/10.1016/S1352-2310(99)00144-2. 